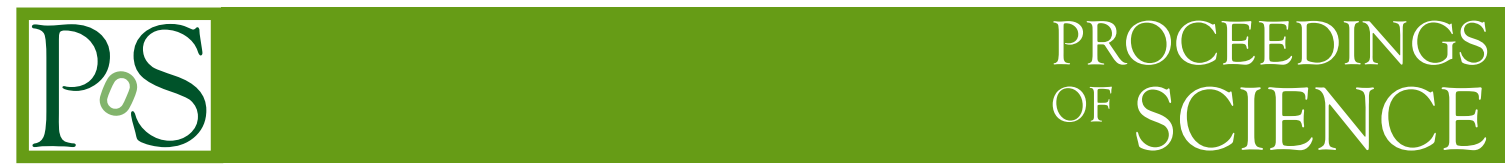

\title{
Asymmetric dark matter and effective number of neutrinos
}

\author{
Yoshihiro KUROSAWA* and Teruyuki KITABAYASHI \\ Department of Physics, Tokai University \\ 4-1-1 Kitakaname, Hiratsuka, Kanagawa, 259-1292, Japan \\ E-mail: 4bsnm002@mail.tokai-u.jp, teruyukietokai-u.jp
}

\begin{abstract}
We study the effect of the $\mathrm{MeV}$-scale asymmetric dark matter annihilation on the effective number of neutrinos $N_{\text {eff }}$ at the epoch of the big bang nucleosynthesis. If the asymmetric dark matter $\chi$ couples more strongly to the neutrinos $v$ than to the photons $\gamma$ and electrons $e^{-}, \Gamma_{\chi \gamma, \chi e} \ll \Gamma_{\chi v}$, or $\Gamma_{\chi \gamma, \chi e} \gg \Gamma_{\chi v}$, the lower mass limit on the asymmetric dark matter is about $18 \mathrm{MeV}$ for $N_{\text {eff }} \simeq 3.0$.
\end{abstract}

The 11th International Workshop Dark Side of the Universe 2015

14-18 December 2015

Kyoto, Japan

\footnotetext{
* Speaker.
} 


\section{Introduction}

The natural scale for asymmetric dark matter (ADM) [W] is around $5 \mathrm{GeV}$ []] , however, mass of

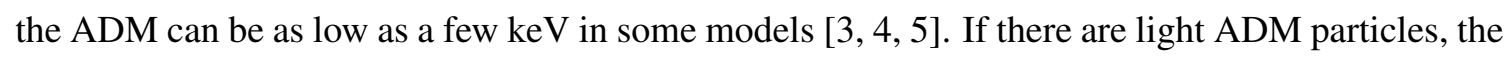
energy density of radiation at big bang nucleosynthesis (BBN) epoch could be changed [ [G, $\square$, , 8$]$ ]. The energy density of the relativistic particles at BBN (as well as at time of cosmic microwave background (CMB) photons released) is usually expressed in terms of the effective number of neutrinos $N_{\text {eff. }}$ By the simple estimation in the standard particle cosmology, we have $N_{v}^{\text {std }}=3$ (more precisely, $N_{v}^{\text {std }}=3.046$ [囫). The effective number of neutrino can be probed by its effect on the CMB $\left(N_{\text {eff }}=3.04 \pm 0.18\right.$ (68\% C.L. $\left.)[\square]\right)$ and the outcome of BBN $\left(N_{\text {eff }}=3.71_{-0.45}^{+0.47}\right.$ [可]).

The extra symmetric dark matter (DM) particles at BBN contribute to the effective number of neutrinos in the following two cases:

1. Direct contribution case: If an extra particle is light enough, it is regarded as one of the radiation components. This light particle has contributed directly to the effective number of neutrinos as the so-called dark radiation [ए], ए2].

2. Indirect contribution case: Although the extra particles are not light enough to contribute directly to the effective number of neutrinos, its annihilation heats other particles via entropy transfer. Either increase or decrease of the effective number of neutrinos occurs as follows:

(i) If an extra particle couples more strongly to the neutrinos $v$ than to the photons $\gamma$ and electrons $e^{-}, \Gamma_{\chi \gamma, \chi e} \ll \Gamma_{\chi v}$, its late time annihilation heats the neutrinos more than the photons. Ultimately, this type of extra particle yields an excess of the effective number of neutrinos,

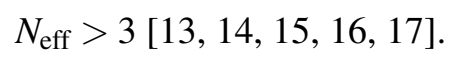

(ii) On the contrary, if an extra particle couples more strongly to the electrons and photons than to the neutrinos, $\Gamma_{\chi \gamma, \chi e} \gg \Gamma_{\chi v}$, its annihilation heats the electron-photon plasma relative to the neutrino background, leading to a reduction in the effective number of neutrinos below

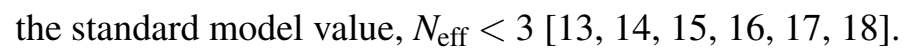

In this paper (this paper is based on Ref.[प्प]), the known method to estimate the effective number of neutrinos with light extra symmetric DM by Steigman in the indirect contribution case [ए5] is extended to the light asymmetric DM in a straightforward way. A lower limit on the ADM mass is obtained.

\section{Asymmetric dark matter and effective number of neutrinos}

\subsection{Asymmetry and chemical potential}

The number asymmetry of ADM is obtained as $\varepsilon=\left(n_{\chi}-n_{\bar{\chi}}\right) / s$. The number density $n_{i}$, energy density $\rho_{i}$, pressure $P_{i}$ and entropy density $s_{i}$ of particle species $i$ with mass $m_{i}$ in the isotropic universe are obtained as follows [[D]]:

$$
\begin{aligned}
& n_{i}=\frac{1}{2 \pi^{2}} \int_{m_{i}}^{\infty} E\left(E^{2}-m_{i}^{2}\right)^{1 / 2} f_{i} d E, \quad \rho_{i}=\frac{1}{2 \pi^{2}} \int_{m_{i}}^{\infty} E^{2}\left(E^{2}-m_{i}^{2}\right)^{1 / 2} f_{i} d E, \\
& P_{i}=\frac{1}{6 \pi^{2}} \int_{m_{i}}^{\infty}\left(E^{2}-m_{i}^{2}\right)^{3 / 2} f_{i} d E, \quad s_{i}=\frac{\rho_{i}+P_{i}-\mu_{i} n_{i}}{T_{i}} .
\end{aligned}
$$


with the distribution function

$$
f_{i}=\frac{g_{i}}{e^{\left(E_{i}-\mu_{i}\right) / T_{i}}+1},
$$

where $g_{i}, E_{i}, \mu_{i}$ and $T_{i}$ denote number of internal degrees of freedom, energy, chemical potential and temperature, respectively. In the ADM scenarios, the ADM particle $\chi$ and its antiparticle $\bar{\chi}$ have nonzero chemical potentials and the particle $\chi$ is not self-conjugate of the antiparticle $\bar{\chi}$. To study the dependence of the light ADM on the effective number neutrinos, we should take care of the chemical potential of ADM. The ratio $\xi=\mu_{\chi} / T_{\chi}$ is the so-called degeneracy parameter or the pseudochemical potential. In the remaining part of this paper, we call $\xi$ chemical potential simply.

\subsection{Effective number of neutrinos}

The energy density of relativistic particles is given by

$$
\rho_{\mathrm{rad}}=\rho_{\gamma}+\rho_{v}^{\mathrm{std}}+\rho_{\mathrm{DR}},
$$

where $\rho_{\gamma}$ is the energy density of photons and $\rho_{v}^{\text {std }}$ is the energy density of standard-model massless neutrinos for $N_{v}^{\text {std }}$ neutrino families.

The energy density of extra radiation, dark radiation, is generally parametrized through the number of extra effective neutrino species $\Delta N_{v}$ as follows [8]

$$
\rho_{\mathrm{DR}}=\Delta N_{v} \rho_{v}=\Delta N_{v} \frac{7 \pi^{2}}{120} T_{v}^{4} .
$$

In terms of the following effective number neutrinos

$$
N_{\text {eff }}=N_{v}^{\mathrm{std}}+\Delta N_{v}
$$

the total energy density of radiation components is estimated to be

$$
\rho_{\text {rad }}=\rho_{\gamma}+N_{\text {eff }} \rho_{v}=\left[1+N_{\text {eff }} \frac{7}{8} \frac{g_{v}}{g_{\gamma}}\left(\frac{T_{v}}{T_{\gamma}}\right)^{4}\right] \rho_{\gamma} .
$$

As we mentioned in the introduction, although the extra particles are not light enough to be a dark radiation, its annihilation heats other particles via entropy conservation and changes the ratio of $\left(T_{v} / T_{\gamma}\right)$ in Eq.(2.6). Consequently, the change of $\rho_{\text {rad }}$ reflects to the enhance or the reduce of the effective number of neutrinos $N_{\text {eff }}$ even in the absence of dark radiation.

\subsection{ADM and $N_{\text {eff }}$}

Symmetric DM case (i) $\Gamma_{\chi \gamma, \chi e} \ll \Gamma_{\chi v}$ : If the symmetric MeV DM $\chi$ couples more strongly to the neutrinos $v$ than to the photons $\gamma$ and electrons $e^{-}$, e.g. $\Gamma_{\chi \gamma, \chi e} \ll \Gamma_{\chi v}$, its late time annihilation heats the neutrinos more than the photons while the annihilation of the $e^{ \pm}$pairs heats the photons (but not the decoupled neutrinos). The ratio of neutrino to photon temperatures after both of the $e^{ \pm}$and the symmetric MeV DM are obtained by consideration of entropy conservation as follows [ए5]]:

$$
\left(\frac{T_{v}}{T_{\gamma}}\right)^{3}=\frac{g_{\gamma}\left[1+\left(\tilde{g}_{\chi} / \tilde{g}_{v}\right)\left(\phi_{\chi} / \phi_{v}\right)\right]}{g_{\gamma}+\tilde{g}_{e} \phi_{e}}=\frac{1+\frac{4}{21} \tilde{g}_{\chi} \phi_{\chi}}{1+\frac{7}{4} \phi_{e}},
$$


where $\tilde{g}_{i}$ denotes effective internal degrees of freedom of the particle $i ; \tilde{g}_{i}=(7 / 8) \cdot 2 \cdot 2=7 / 2$ for the Dirac fermions, $\tilde{g}_{i}=(7 / 8) \cdot 2 \cdot 1=7 / 4$ for the Majorana fermions. Although we consider the only fermionic Dirac type DM, we can also use $\tilde{g}_{i}=1$ for the scalar bosons and 3 for the vector bosons with the bosonic distribution function. The function of $\phi$ denotes the normalized entropy density

$$
\phi_{\alpha}(x)=\frac{s_{\alpha}^{\text {net }}(x)}{s_{\alpha}^{\text {net }}(0)},
$$

where $s_{\alpha}^{\text {net }}(x)$ is the net entropy density of particle species $\alpha$ with vanishing chemical potential:

$$
s_{\alpha}^{\mathrm{net}}(x)=s_{\alpha}\left(\mu_{\alpha}=0\right)+s_{\bar{\alpha}}\left(\mu_{\bar{\alpha}}=0\right)=\sum_{i=\alpha, \bar{\alpha}} \frac{\rho_{i}+P_{i}}{T_{i}} .
$$

For $e^{ \pm}$pairs, $\phi_{e}$ is evaluated at $x=x_{e d}=m_{e} / T_{v d}$, while for the symmetric DM, $\phi_{\chi}$ is evaluated at $x=x_{\chi d}=m_{\chi} / T_{v d}$ where $T_{v d}$ denotes the decoupling temperature of neutrinos. Because the difference between Majorana or Dirac nature of symmetric MeV DM is taken into account by the effective internal degrees of freedom $\tilde{g}_{\chi}$, we take $g_{\chi}=1$ in Eq.(2.2) through our calculations.

With the appropriate assumptions of $T_{v d}=2 \mathrm{MeV}$ and $\phi_{e}=0.993$ [ㄷ]], we obtain

$$
N_{\text {eff }}=3\left[\frac{11}{4}\left(\frac{T_{v}}{T_{\gamma}}\right)^{3}\right]^{4 / 3}=3.018\left(1+\frac{4 \tilde{g}_{\chi} \phi_{\chi}}{21}\right)^{4 / 3}
$$

The effective number of neutrinos is a function of the mass of symmetric MeV DM.

Symmetric DM case (ii) $\Gamma_{\chi \gamma, \chi_{e}} \gg \Gamma_{\chi_{\nu}}$ : On the other hand, if the symmetric MeV DM couples more strongly to the electrons and photons than to the neutrinos, $\Gamma_{\chi \gamma, \chi e} \gg \Gamma_{\chi v}$, its annihilation heats the electron-photon plasma relative to the neutrino background. By the consideration of entropy conservation, we have [ㄷ]]

$$
\left(\frac{T_{v}}{T_{\gamma}}\right)^{3}=\frac{g_{\gamma}}{g_{\gamma}+\tilde{g}_{e} \phi_{e}+\tilde{g}_{\chi} \phi_{\chi}}=\frac{2}{2+\frac{7}{2} \phi_{e}+\tilde{g}_{\chi} \phi_{\chi}},
$$

and

$$
N_{\text {eff }}=3\left[\frac{11}{4}\left(\frac{T_{v}}{T_{\gamma}}\right)^{3}\right]^{4 / 3}=3\left(\frac{11}{10.95+2 \tilde{g}_{\chi} \phi_{\chi}}\right)^{4 / 3} .
$$

ADM case: To take care of the chemical potential of the ADM for the entropy calculations, we use the following net entropy density of the ADM

$$
s_{\chi}^{\mathrm{net}}\left(x, \mu_{\chi}\right)=s_{\chi}+s_{\bar{\chi}}=\sum_{i=\chi, \bar{\chi}} \frac{\rho_{i}+P_{i}-\mu_{i} n_{i}}{T_{i}}
$$

instead of Eq.(2.9). The normalized entropy density of the ADM to be a function of not only $x_{\chi d}$ but also $\mu_{\chi}$ as follows:

$$
\phi_{\chi}\left(x_{\chi d}, \mu_{\chi}\right)=\frac{s_{\chi}^{\mathrm{net}}\left(x_{\chi d}, \mu_{\chi}\right)}{s_{\chi}^{\mathrm{net}}\left(0, \mu_{\chi}\right)}
$$



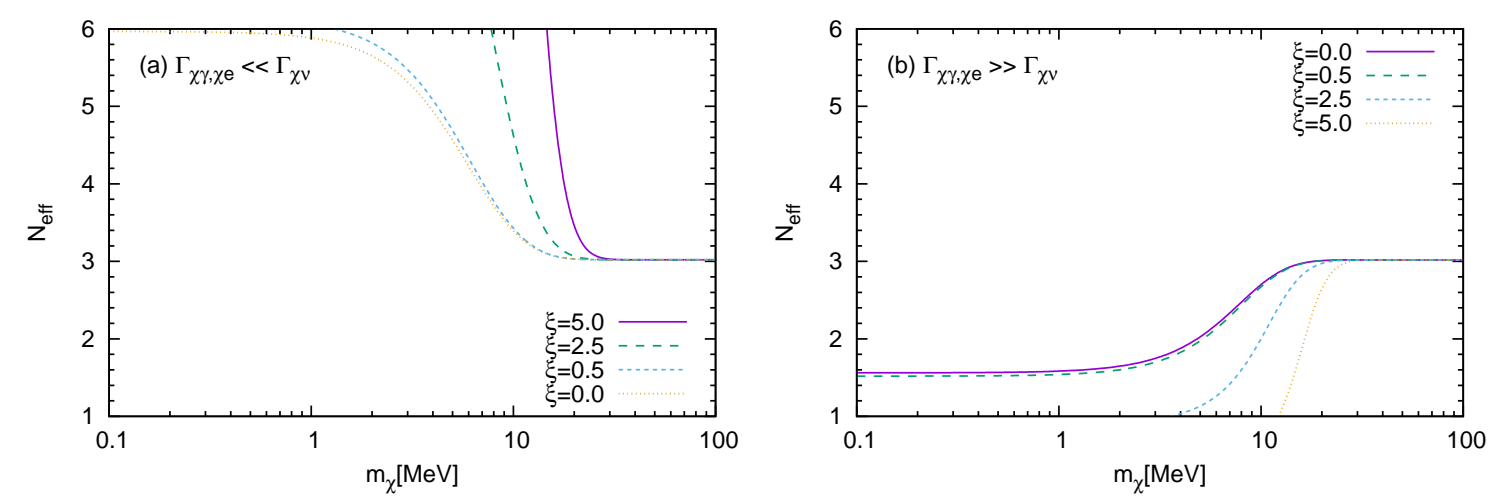

Figure 1: The dependence of the effective number of neutrinos $N_{\text {eff }}$ on the ADM mass $m_{\chi} \mathrm{MeV}$ with the various chemical potential $\xi$ at BBN. The figure (a) shows the dependence in the case of $\Gamma_{\chi \gamma, \chi e} \ll \Gamma_{\chi^{v}}$ while the figure (b) shows the dependence in the case of $\Gamma_{\chi \gamma, \chi e} \gg \Gamma_{\chi v}$.

Other quantities in Eq.(․ㄱ) and Eq.([2.1]), such as $\tilde{g}_{\chi}, \phi_{e}$, remain the same.

The effective number of neutrinos with the MeV ADM is estimated in the same form of Eq.(2.J) and Eq.(2.J2). If the MeV ADM $\chi$ couples more strongly to the neutrinos $v$ than to the photons $\gamma$ and electrons $e^{-}\left(\Gamma_{\chi \gamma, \chi e} \ll \Gamma_{\chi v}\right)$, we obtain

$$
N_{\text {eff }}=3.018\left(1+\frac{4 \tilde{g}_{\chi} \phi_{\chi}\left(x_{\chi d}, \mu_{\chi}\right)}{21}\right)^{4 / 3},
$$

while if the MeV ADM couples more strongly to the electrons and photons than to the neutrinos $\left(\Gamma_{\chi \gamma, \chi e} \gg \Gamma_{\chi v}\right)$, we have

$$
N_{\text {eff }}=3\left(\frac{11}{10.95+2 \tilde{g}_{\chi} \phi_{\chi}\left(x_{\chi d}, \mu_{\chi}\right)}\right)^{4 / 3} .
$$

Figure $\mathbb{W}$ shows the dependence of the effective number of neutrinos $N_{\text {eff }}$ on the mass of the $\operatorname{ADM} m_{\chi}$ with the various chemical potentials $\xi$ at BBN $(T=2 \mathrm{MeV})$. The figure (a) shows the dependence in the case of $\Gamma_{\chi \gamma, \chi e} \ll \Gamma_{\chi^{v}}$ while the figure (b) shows the dependence in the case of $\Gamma_{\chi \gamma, \chi e} \gg \Gamma_{\chi v}$.

The similar figure of Fig.W has already reported by Nollett and Steigman for symmetric DM [ए6, [7] . The curves with nonvanishing chemical potential in Fig. 1 are newly obtained in our study. Compare with the symmetric DM case, the effective number of neutrinos $N_{\text {eff }}$ increases with the increasing asymmetry (chemical potential $\xi$ ) and with the decreasing mass $m_{\chi}$ if ADM particle mainly interacts to neutrinos $\left(\Gamma_{\chi \gamma, \chi e} \ll \Gamma_{\chi v}\right)$. On the contrary, $N_{\text {eff }}$ decreases with the increasing $\xi$ and with the decreasing $m_{\chi}$ if ADM particle mainly interacts to photons and electrons $\left(\Gamma_{\chi \gamma, \chi e} \gg \Gamma_{\chi v}\right)$. Also, in the Nollett and Steigman papers for symmetric DM [ए6, [1]], $N_{\text {eff }}$ depends on the nature of the quantum statistics of thermal relic (i.e., fermion or boson). We can take the effective internal degrees of freedom as $\tilde{g}_{\chi}=1,7 / 4,2,7 / 2$ for a real scalar, Majorana fermion, complex scalar and Dirac fermion in Eqs([2.15) and ([2.16). Thus, $N_{\text {eff }}$ depends on the difference of the nature of the thermal relic not only in the symmetric DM case but also in the ADM case. 

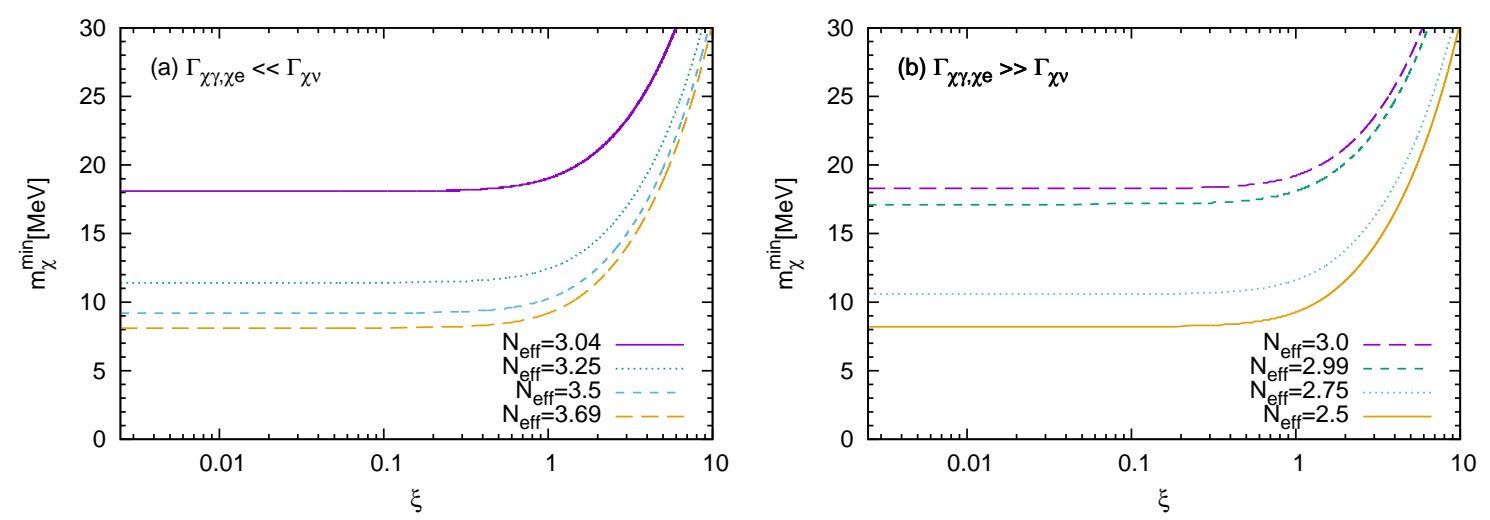

Figure 2: The dependence of the lower mass limit of the ADM $m_{\chi}^{\min }$ on the chemical potential $\xi$ with the various effective number of neutrinos $N_{\text {eff }}$ at $\mathrm{BBN}(T=2 \mathrm{MeV})$. The figure (a) shows the $\Gamma_{\chi \gamma, \chi e} \ll \Gamma_{\chi v}$ case while the figure (b) shows the $\Gamma_{\chi \gamma, \chi e} \gg \Gamma_{\chi v}$ case.

The lower mass limit is obtained with the upper bound or lower bound on the effective number of neutrinos. The lower bound on the ADM mass is the case when the asymmetry and chemical potential are small. The bound gets stronger as asymmetry $\varepsilon$ and chemical potential $\xi$ grow. It shows the smooth transition from asymmetric to the symmetric WIMP limit.

Fig.】 shows the dependence of the lower mass limit of the $\mathrm{ADM} m_{\chi}^{\min }$ on the chemical potential $\xi$ with the various bound of the effective number of neutrinos $N_{\text {eff }}$ at BBN. The figure (a) shows the $\Gamma_{\chi \gamma, \chi e} \ll \Gamma_{\chi^{v}}$ case while the figure (b) shows the $\Gamma_{\chi \gamma, \chi_{e}} \gg \Gamma_{\chi^{v}}$ case. From Fig.】, the following constraint on $m_{\chi}^{\min }$ with $N_{\text {eff }}$ is obtained:

$$
m_{\chi}^{\min } \simeq \begin{cases}8.1-18.1 \mathrm{MeV} & \left(N_{\mathrm{eff}}^{\max }=3.0-3.7\right) \\ 8.2-18.3 \mathrm{MeV} & \left(N_{\mathrm{eff}}^{\min }=2.5-3.0\right)\end{cases}
$$

for $\Gamma_{\chi \gamma, \chi e} \ll \Gamma_{\chi v}$ (upper) and $\Gamma_{\chi \gamma, \chi e} \gg \Gamma_{\chi v}$ (lower) cases, more concretely,

$$
m_{\chi}^{\min } \simeq \begin{cases}18.1 \mathrm{MeV} & \left(\Gamma_{\chi \gamma, \chi e} \ll \Gamma_{\chi v}, N_{\mathrm{eff}}^{\max }=3.0\right) \\ 18.3 \mathrm{MeV} & \left(\Gamma_{\chi \gamma, \chi e} \gg \Gamma_{\chi v}, N_{\mathrm{eff}}^{\min }=3.0\right)\end{cases}
$$

with the standard value of the effective number of neutrinos.

\section{Summary}

We have extended the known method by Steigman [45] to obtain the constraints on the $\mathrm{MeV}$ asymmetric dark matter with the effective number of neutrinos $N_{\text {eff }}$ at big bang nucleosynthesis. If the $\mathrm{MeV}$ asymmetric dark matter couples more strongly to the neutrinos than to the photons and electrons $\left(\Gamma_{\chi \gamma, \chi e} \ll \Gamma_{\chi v}\right)$ or if the $\mathrm{MeV}$ asymmetric dark matter couples more strongly to the electrons and photons than to the neutrinos $\left(\Gamma_{\chi \gamma, \chi e} \gg \Gamma_{\chi v}\right)$, the constraint on $m_{\chi}^{\min }$ with $N_{\text {eff }}$ is obtained as $m_{\chi}^{\min } \simeq 18 \mathrm{MeV}$ for $N_{\text {eff }}=3.0$.

From the galactic $511 \mathrm{keV}$ gamma-ray line observation, the symmetric dark matter mass may be less than about a few $10 \mathrm{MeV}$ (or a few MeV) [2], [2]]. We can expect that the range of the 
asymmetric (as well as symmetric) $\mathrm{MeV}$ dark matter mass is so narrow to satisfy $m_{\chi} \simeq 18 \mathrm{MeV}$ in the case of $\Gamma_{\chi \gamma, \chi e} \gg \Gamma_{\chi v}$ or $\Gamma_{\chi \gamma, \chi e} \ll \Gamma_{\chi v}$.

\section{References}

[1] K. M. Zurek, Asymmetric Dark Matter: Theories, signatures, and constraints, Phys. Rep. 537, 91 (2014).

[2] G. Gelmini, L. I. Hall and M. Lin, What is the cosmion?, Nucl. Phys. B 281, 726 (1987).

[3] A. Falkowski, J. T. Ruderman and T. Volansky, Asymmetric dark matter from leptogenesis, JHEP 05, 106 (2011).

[4] R. T. D'Agnolo and A. Hook, Spontaneous baryogenesis from extremely light asymmetric dark matter, Phys. Rev. D 91, 115020 (2015).

[5] E. Izaguirre, G. Krnjaic and M. Pospelov, MeV-scale dark matter deep underground, Phys. Rev. D 92 , 095014 (2015).

[6] G. Steigman, Neutrinos and Big Bang Nucleosynthesis, Adv. High Energy Phys. id:268321 (2012).

[7] S. R.-Sørensen, D. Parkinson and T. M. Davis, What Is Half a Neutrino? Reviewing Cosmological Constraints on Neutrinos and Dark Radiation, PASA 30, e029 (2013).

[8] J. Lesgourgues, G. Mangano, G. Miele and S. Pastor, Neutrino Cosmology, Cambridge University Press (2013).

[9] G. Mangano, G. Miele, S. Pastor, T. Pinto, O. Pisanti and P. D. Serpico,Relic neutrino decoupling including flavour oscillations, Nucl. Phys. B 729, 221 (2005).

[10] P. A. R. Ade, et. al. (Planck Collaboration), Planck 2015 results. XIII. Cosmological parameters, arXiv:1502.01589v2 (Feb,2015).

[11] C. M. Ho and R. J. Scherrer, Sterile neutrinos and light dark matter save each other, Phys. Rev. D 87, 065016 (2013).

[12] T. D. Jacques, L. M. Krauss and C. Lunardini, Additional light sterile neutrinos and cosmology, Phys. Rev. D 87, 083515 (2013).

[13] E. W. Kolb, M. S. Turner and T. P. Walker, Effect of interacting particles on primordial nucleosynthesis, Phys. Rev. D 34, 2197 (1986).

[14] P. D. Serpico and G. G. Raffelt, MeV-mass dark matter and primordial nucleosynthesis, Phys. Rev. D 70, 043526 (2004).

[15] G. Steigman, Equivalent neutrinos, light WIMPs, and the chimera of dark radiation, Phys. Rev. D 87, 103517 (2013).

[16] K. M. Nollett and G. Steigman, BBN and the CMB constrain light, electromagnetically coupled WIMPs, Phys. Rev. D 89, 083508 (2014).

[17] K. M. Nollett and G. Steigman, BBN and the CMB constrain neutrino coupled light WIMPs, Phys. Rev. D 91, 083505 (2015).

[18] C. M. Ho and R. J. Scherrer, Limits on MeV dark matter from the effective number of neutrinos, Phys. Rev. D 87, 023505 (2013).

[19] T. Kitabayashi and Y. Kurosawa, Asymmetric dark matter and effective number of neutrinos, Phys. Rev. D 93, 033002 (2016). 
[20] E. W. Kolb and M. S. Turner, The Early Universe, Addison-Wesley (1990).

[21] J. F. Beacom and H. Yüksel, Stringent Constraint on Galactic Positron Production, Phys. Rev. Lett. 97, 071102 (2006).

[22] P. Sizun, M Cassé and S. Schanne, Continuum $\gamma$-ray emission from light dark matter positrons and electrons,Phys. Rev. D 74, 063514 (2006). 